\title{
Radiation induces osteogenesis in human aortic valve interstitial cells
}

\author{
Nicole A. Nadlonek, MD, Michael J. Weyant, MD, Jessica A. Yu, MD, Joseph C. Cleveland, Jr, MD, \\ T. Brett Reece, MD, Xianzhong Meng, MD, PhD, and David A. Fullerton, MD
}

\begin{abstract}
Objective: Irradiation of the chest or chest wall has been shown to cause calcific aortic stenosis. However, the mechanisms are unknown. Aortic valve interstitial cells have been implicated in the pathogenesis of aortic stenosis; they have been shown to change from the phenotype of a myofibroblast to an osteoblastlike cell. We therefore hypothesized that irradiation of human aortic valve interstitial cells induces an osteogenic phenotype. In isolated human aortic valve interstitial cells, our purpose was to determine the effect of irradiation on the production of osteogenic factors: (1) bone morphogenetic protein 2, (2) osteopontin, (3) alkaline phosphatase, and
\end{abstract} (4) the transcription factor Runx2.

\begin{abstract}
Methods: Human aortic valve interstitial cells were isolated from normal aortic valves obtained from explanted hearts of patients undergoing cardiac transplantation $(n=4)$ and were grown in culture. The cells were grown to confluence, irradiated with 10 Gy using a cesium-137 irradiator, and then lysed 24 hours after irradiation. Cell lysates were analyzed via immunoblot and densitometry for bone morphogenetic protein 2, osteopontin, alkaline phosphatase, and Runx2. Statistical analysis was performed using analysis of variance, with $P<.05$ indicating significance.
\end{abstract}

Results: Irradiation induced an osteogenic phenotype in human aortic valve interstitial cells. Irradiation induced a 2-fold increase in bone morphogenetic protein 2, a 7-fold increase in osteopontin, a 3-fold increase in alkaline phosphatase, and a 2-fold increase in Runx2.

Conclusions: Radiation induces an osteogenic phenotype in human aortic valve interstitial cells. The irradiated cells had a significantly increased expression of the osteogenic factors bone morphogenetic protein 2 , osteopontin, alkaline phosphatase, and Runx2. These data offer mechanistic insight into the pathogenesis of radiationinduced valvular heart disease. (J Thorac Cardiovasc Surg 2012;144:1466-70)

The use of radiation to treat malignancies of the chest and chest wall is associated with increased rates of cardiac disease, including valvular disease. The majority of these valvular defects involve the aortic and mitral valves. ${ }^{1-3}$ Within 20 years following mantle radiation, as many as $16 \%$ of patients will develop calcific aortic stenosis; this is at least 3 times the incidence found in the general population. ${ }^{4}$ This is particularly significant because many patients who undergo chest radiation therapy are young adults, leading to the development of aortic stenosis at a younger age than commonly seen. In fact, among patients with aortic stenosis, those with a prior history of mantle irradiation develop aortic stenosis at an age that is approximately 20 years younger than those patients with no prior

From the Division of Cardiothoracic Surgery, University of Colorado School of Medicine, Aurora, Colo.

Supported in part by American Heart Association grant 11GRNT7900016 and National Institutes of Heart, Lung, and Blood grant HL106582.

Disclosures: Authors have nothing to disclose with regard to commercial support.

Read at the 38th Annual Meeting of The Western Thoracic Surgical Association, Maui, Hawaii, June 27-30, 2012.

Received for publication June 22, 2012; revisions received Aug 1, 2012; accepted for publication Aug 16, 2012; available ahead of print Oct 1, 2012.

Address for reprints: David A. Fullerton, MD, Cardiothoracic Surgery, 12631 E. 17th Ave, MS C-310, Room 6602, Aurora, CO 80045 (E-mail: David.Fullerton@ UCDenver.edu)

0022-5223/\$36.00

Copyright (c) 2012 by The American Association for Thoracic Surgery

http://dx.doi.org/10.1016/j.jtcvs.2012.08.041 irradiation. $^{2,5}$ Furthermore, following chest radiation therapy, the risks of cardiac surgical operations, including aortic valve replacement, are significantly increased. ${ }^{6-8}$

The mechanisms of radiation-induced aortic stenosis are unknown. However, in other tissues radiation has been shown to be inflammatory. The principal cell type found in the human aortic valve, the aortic valve interstitial cell (AVIC), has been implicated in the pathogenesis of calcific aortic stenosis. When stimulated by proinflammatory stimuli, isolated human AVICs have been shown to undergo a change in phenotype from that of a myofibroblast to that of an osteoblastlike cell. ${ }^{9,10}$ This osteogenic phenotype is characterized by the production of osteogenic factors such as bone morphogenetic protein 2 (BMP-2; a protein necessary for bone formation), osteopontin (OPN; an important bone remodeling protein), alkaline phosphatase (ALP; an enzyme important for bone mineralization), and the transcription factor Runx2, which is necessary for bone formation.

Given the recognized proinflammatory actions of radiation, we hypothesized that radiation-induced aortic stenosis derives from the induction of an osteogenic phenotype in human AVICs. The purpose of this study was to determine the effect of irradiation on the production of BMP-2, OPN, ALP, and Runx2 in isolated human AVICs. The results of this study demonstrate that radiation induces an osteogenic phenotype in human AVICs. 

Abbreviations and Acronyms
ALP = alkaline phosphatase
AVIC $=$ aortic valve interstitial cell
BMP-2 = bone morphogenetic protein 2
OPN = osteopontin
PBS = phosphate-buffered saline
T-PBS $=$ Tween in phosphate-buffered saline

\section{MATERIALS AND METHODS}

This study was approved by the Colorado multiple institutional review board of the University of Colorado School of Medicine. All patients provided written informed consent.

\section{Chemicals and Reagents}

Medium 199 was purchased from Lonza (Walkersville, Md). Rabbit polyclonal antibody against human BMP-2 was purchased from Prosci (Poway, Calif) and rabbit polyclonal antibody against intercellular adhesion molecule 1 was purchased from AbD Serotec (Raleigh, N.C). Protein assay reagents and chemiluminescent substrate (enhanced chemiluminescence) were purchased from Thermo Scientific (Rockford, Ill). Forty to $20 \%$-gradient polyacrylamide ready gels, nitrocellulose membranes, and $2 \times$ Laemmli sample buffer were purchased from Bio-Rad (Hercules, Calif). All other chemicals were purchased from Sigma Chemical Co. (St Louis, Mo).

\section{Cell Isolation and Culture}

Grossly normal aortic valve leaflets $(n=4)$ were obtained from explanted hearts from patients (male, 28-48 years) undergoing cardiac transplantation at the University of Colorado Hospital. On gross examination, all leaflets were thin, pliable, and without calcification. Isolation by collagenase digestion was performed as described previously, ${ }^{9}$ and AVICs were cultured and maintained as independent cultures in medium 199 with penicillin G, streptomycin, amphotericin B, and 10\% fetal bovine serum in an incubator supplied with 5\% carbon dioxide. The aortic valves were placed in $4^{\circ} \mathrm{C}$ sterile saline immediately after removal. The valves were washed with sterile saline 3 times and then sectioned. Segments were placed into $4 \%$ formaldehyde in phosphate-buffered saline (PBS), flash frozen, or placed in tissue-freezing media for frozen sectioning. The remaining sections were washed 5 times with Earl's Balanced Salt Solution, placed in $2.5 \mathrm{mg} / \mathrm{mL}$ collagenase in full medium 199 for 30 minutes, and incubated at $37^{\circ} \mathrm{C}$. After discarding the supernatant, the valve sections were washed once with Earl's Balanced Salt Solution to remove endothelial cells. The valve segments were then placed in $0.8 \mathrm{mg} / \mathrm{mL}$ collagenase in full medium 199 and digested for 3 additional hours. The supernatant from this digestion was centrifuged, the pellet resuspended in full medium 199, and the cells were grown in culture (passage 0). Cells from passages 3 to 6 grown to $70 \%$ to $90 \%$ confluence were used for all experiments.

\section{Radiation}

To irradiate the cells in culture, 24-well plates of cells were first prepared. These were allowed 24 hours of incubation at $37^{\circ} \mathrm{C}$ with $5 \%$ carbon dioxide for 24 hours prior to transport to a cesium-137 irradiator. The control cells were transported to the radiation suite as well, although they were not treated. The experimental cells underwent $10 \mathrm{~Gy}$ total irradiation (175 $\mathrm{rad} / \mathrm{min}$ for 2.85 minutes twice). The cells were then transported back to the incubator until lysis at 24 hours. The cells were checked for morphologic changes prior to lysis, and none were present. The radiation doses were adapted from Williams and colleagues. ${ }^{11}$

\section{Immunofluorescence}

Slides were prepared with normal and stenotic valve specimens from frozen OCT preparations. These slides were dried overnight, then fixed in 30\% acetone: $70 \%$ methanol for 10 minutes. After 3 PBS washes for 10 minutes each, the slides were blocked with $10 \%$ nonimmune donkey serum in PBS for 1 hour. Intercellular adhesion molecule 1 (AbD Serotec, Raleigh, N.C) and BMP-2 primary antibody (Abcam, Cambridge, Mass) was diluted in $1 \%$ bone serum albumin to concentrations of 1:50 and 1:250, respectively. A total of $100 \mu \mathrm{L}$ of this dilution was placed on the mounted tissue and incubated at $4{ }^{\circ} \mathrm{C}$ overnight in a box with a moist towel. Negative controls were prepared as well, with rabbit immunoglobulin $\mathrm{G}$ only in the solution at the same concentrations. The following morning, the slides were washed in PBS 3 times. The secondary antibody solution was then prepared with wheat germ agglutinin (Alexa 633) 1:500, donkey antirabbit (Alexa 488) $1: 100$ and donkey antimouse (Alexa 555) $1: 100$. A total of $100 \mu \mathrm{L}$ of this solution was placed on the mounted tissue and the slides were incubated for 45 minutes at room temperature. After 3 more PBS washes, antiquenching medium containing bisbenzimide (Invitrogen, Grand Island, NY) was placed on the tissue and the coverslips were put in place. The next morning, the slides were sealed and viewed.

Examination of aortic valve leaflets from a patient with prior irradiation. A 56-year-old male with a prior history of mediastinal irradiation underwent heart transplantation at our institution. This event provided the opportunity to examine the aortic valve leaflets from his explanted heart. He had no gradient across the aortic valve on pretransplant echocardiogram. The valve leaflets were grossly normal. The aortic valve leaflets from the explanted heart were examined for BMP-2 expression (immunofluorescence methodology described earlier). For comparison, the leaflets from the explanted, irradiated valve were compared with age-matched aortic valve leaflets from a normal aortic valve (control) and a stenotic aortic valve from a patient undergoing aortic valve replacement for aortic stenosis with no history of irradiation.

\section{Immunoblotting}

Cell lysates were analyzed for BMP-2, OPN, ALP, and Runx2 by immunoblotting. AVICs in culture were lysed in $1 \times$ Laemmli sample buffer with $\beta$-mercaptoethanol. Lysates were loaded into a 15 -well 4 to $20 \%$-gradient ready gels (Bio-Rad) and run at $200 \mathrm{~V}$ for 30 minutes. The gels were transferred to nitrocellulose membranes at $100 \mathrm{~V}$ for 70 minutes, then were cross-linked twice using a UV Stratalinker (Stratagene, La Jolla, Calif). The membranes were blocked in 5\% dry milk in $0.1 \%$ Tween in PBS (T-PBS), then rinsed 3 times in $0.1 \%$ T-PBS. The blocked membranes were incubated with primary antibodies overnight at $4{ }^{\circ} \mathrm{C}$ (diluted to $1: 1000$ to $1: 10,000$ in $5 \%$ bone serum albumin in $0.1 \% \mathrm{~T}$-PBS). The membranes were washed in $0.1 \%$ T-PBS 3 times and then incubated in appropriate horseradish peroxidaseconjugated secondary antibodies diluted to $1: 5000$ in 5\% dry milk in $0.1 \%$ T-PBS for 1 hour at room temperature. After decanting the secondary antibody, the wash step was repeated and the membranes were placed in ECL for 5 minutes at room temperature and exposed on X-ray film. The film was scanned using an Epson flatbed scanner (Long Beach, Calif), and densitometry was performed using Image $\mathbf{J}$ software from the National Institutes of Health (Wayne Rasband, National Institutes of Health, Bethesda, Md).

\section{Statistical Analysis}

Data are represented as mean \pm standard error mean. Statistical analysis was performed using analysis of variance; $P<.05$ was considered significant.

\section{RESULTS}

Increased Expression of BMP-2 in the Aortic Valve of a Patient With Prior Mediastinal Radiation

A 56-year-old male with a prior history of mantle radiation (lymphoma) underwent heart transplantation at our 

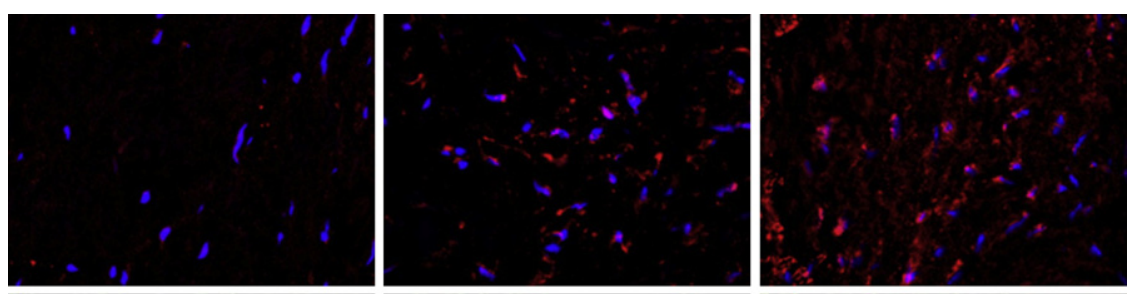

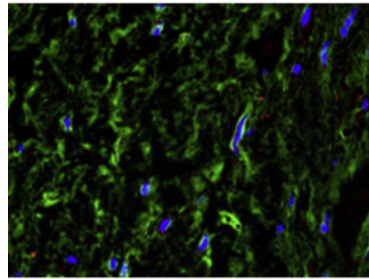

Control

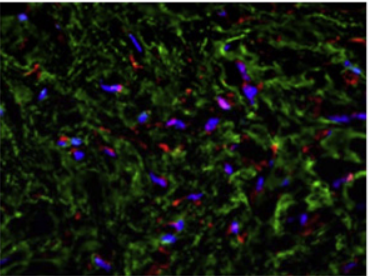

Stenotic

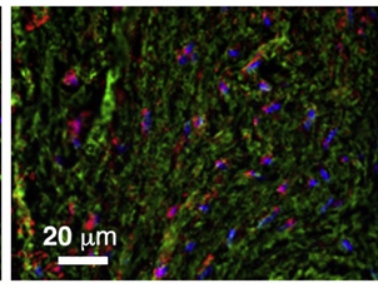

Irradiated

FIGURE 1. Immunofluorescent staining of aortic valve leaflets. The bone morphogenic protein 2 (BMP-2) signal is increased (immunofluorescence) for both stenotic and irradiated aortic valve tissue compared with nonstenotic controls. Upper panels, The red stain is for BMP-2; the blue is for nuclei (bisbenzimide). Lower panels, The green stain is for cellular architecture (WGA). The control and irradiated leaflets were from grossly normal valves. The stenotic leaflet was from a calcified aortic valve removed at aortic valve replacement. Magnification, $40 \times$.

institution. Although the aortic valve leaflets from his explanted heart were grossly normal, histologic examination revealed the increased presence of BMP-2. As demonstrated in Figure 1, immunofluorescence staining revealed no evidence of BMP-2 in normal valve leaflets (control). By comparison, leaflets from a patient with calcific aortic stenosis (no history of radiation) demonstrated increased BMP-2 expression. The leaflets from this patient with a history of prior radiation demonstrated significantly increased BMP-2 expression.

\section{Radiation-Induced Increased Expression of BMP-2, OPN, ALP, and Runx in Human AVICs}

As shown in Figure 2, BMP-2 expression was increased significantly (2-fold, $P<.05$ ) after irradiation. Not only was the protein expression increased significantly but also BMP-2 levels in the cell culture media were increased significantly-2-fold $(P<.05)$. This is noteworthy because once synthesized within the cell, BMP-2 is secreted by the cell and acts in an autocrine and paracrine fashion. These data indicate that BMP-2 secretion by the AVICs was increased after radiation.

Other factors associated with osteogenesis were also increased after irradiation. As shown in Figure 3, OPN, ALP, and Runx2 were all increased significantly. Twentyfour hours after irradiation, OPN expression was increased $>7$-fold in comparison with cells that were not irradiated $(P<.05)$. The expression of ALP was increased nearly 3 -fold $(P<.05)$. The expression of the transcription factor, Runx2, was increased 2-fold after irradiation $(P<.05)$. Cells that were not removed from the lab showed no difference in comparison with control cells taken to the radiation suite (data not shown).

\section{DISCUSSION}

Radiation-induced valvular disease, particularly aortic stenosis, affects a substantial number of the patients treated with chest radiation. ${ }^{12,13}$ The results of the current study demonstrate that irradiation of human AVICs induces an osteogenic phenotype with increased production of the osteogenic factors BMP-2, OPN, ALP, and transcription factor Runx2. These data offer mechanistic insight into the pathogenesis of radiation-induced calcific aortic stenosis.

In the current study, the effects of radiation were examined in vitro. Others have found that the effects of radiation are even more pronounced in cells in vivo than in culture. ${ }^{14}$ Such data suggest that the in vivo cellular environment and cell-cell interactions in vivo may augment the effects of irradiation. In addition, only 1 dose of radiation was used; it was chosen from an optimization done for cells in culture that does not affect viability. ${ }^{11}$ It must be acknowledged that different radiation dosages may have other effects. Despite these limitations, however, the results of the current study demonstrate that radiation induces an osteogenic phenotype in isolated human AVICs. Furthermore, the finding of significantly increased expression of BMP-2 in the aortic valve leaflets from a patient with prior mantel irradiation (Figure 1) lends strong support to the in vitro findings of the current study.

Irradiation of isolated cells has been shown previously to have proinflammatory effects. Jelonek and colleagues ${ }^{15}$ recently examined the effects of radiation on mouse endothelial cells in vitro and in vivo, and demonstrated increased expression of endothelial-specific inflammatory and cell adhesion molecules after irradiation. Ryu and associates ${ }^{16}$ found that mitogen-activated protein kinases, such as c-jun terminal kinase, extracellular related kinases, and p38, are upregulated in radiation-induced pneumonitis in 

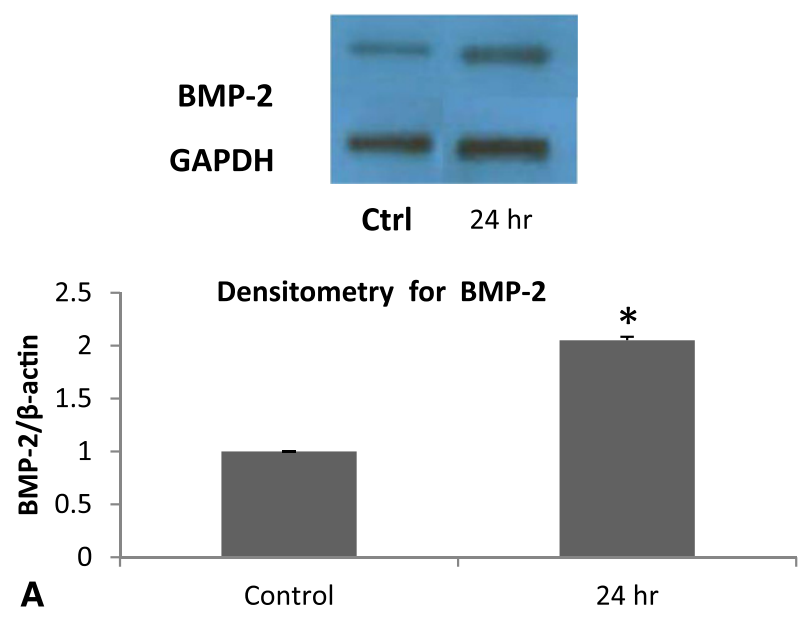

ELISA for BMP-2

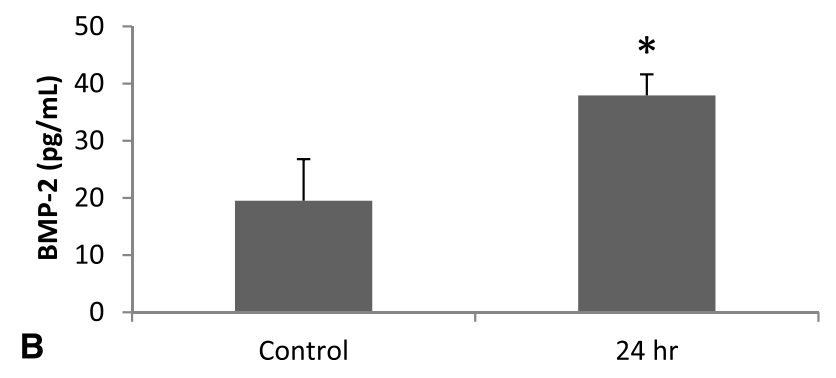

FIGURE 2. Bone morphogenic protein $2(B M P-2)$ expression was increased in irradiated human aortic valve interstitial cells. A, Representative immunoblot and densitometry shows increased expression in cell lysate 24 hours after irradiation. B, The concentration of BMP-2 is increased 24 hours after irradiation in cell culture media. $* P<.05$. Ctrl, Control; $G A P D H$, glyceraldehyde phosphate dehydrogenase; ELISA, enzymelinked immunosorbent assay.

rats. These intracellular signaling cascades have been implicated in the pathogenesis of aortic stenosis, ${ }^{17}$ and are an example of the mechanistic linkage between inflammation and radiation-induced damage. ${ }^{18}$

To our knowledge, this is the first study to examine the effects of radiation on isolated human AVICs. Work from our laboratory has previously demonstrated that proinflammatory stimulation may induce an osteogenic phenotype in human AVICs. ${ }^{9,10,16}$ Given the proinflammatory effects of irradiation, ${ }^{15}$ it seems possible that the mechanisms by which radiation induces an osteogenic phenotype in human AVICs may involve mechanisms of inflammation.

Cardiac valve disease is a particularly vexing complication of chest radiation therapy. Radiation-associated cardiac valve disease has been described after irradiation of the breast, lung, and chest wall, but it is particularly prevalent after mediastinal irradiation. As many as $60 \%$ of patients develop radiation-associated cardiac valve pathology. ${ }^{1}$ The increased risks of cardiac surgical operations in patients with a history of prior mediastinal radiation are well-recognized. ${ }^{6,7}$ The risks of aortic valve replacement may be increased after mediastinal irradiation, in part, because the effects of radiation are not confined to the valve. Although probably dose dependent, mediastinal irradiation may result in significant mediastinal fibrosis along with constrictive pericardial disease, restrictive myocardial disease, coronary artery disease, and aortic fibrosis. These injuries may culminate in increased cardiac surgical risks in patients with prior mediastinal irradiation. Hence, a greater understanding of the mechanisms responsible for radiation-induced aortic stenosis may be an important step in preventing this complication of chest radiation therapy.

In summary, the results of the current study demonstrate that radiation induces an osteogenic phenotype in human AVICs irradiated in vitro. These findings offer mechanistic


FIGURE 3. The expression of osteopontin $(O P N)$, alkaline phosphatase $(A L P)$, and Runx 2 were increased after radiation. Using immunoblotting and densitometry, expression of OPN, ALP, and Runx 2 was increased 24 hours after irradiation. ${ }^{*} P<.05$. GAPDH, glyceraldehyde phosphate dehydrogenase; $C t r l$, control. 
insight into the pathogenesis of radiation-induced calcific aortic stenosis.

\section{References}

1. Heidenriech PA, Hancock SL, Lee BK, Mariscal CS, Schnittger I. Asymptomatic cardiac disease following mediastinal irradiation. J Am Coll Cardiol. 2003;42: 743-9.

2. Wethal T, Lund W-B, Edvardsen T, Fossa SD, Pripp AH, Holte H, et al. Valvular dysfunction and left ventricular changes in Hodgkin's lymphoma survivors. A longitudinal study. Br J Cancer. 2009;101:575-81.

3. Cella L, Liuzzi R, Conson M, Torre G, Caterino M, De Rosa N, et al. Dosimetric predictors of asymptomatic heart valvular dysfunction following mediastinal irradiation for Hodgkins lymphoma. Radiother Oncol. 2011;101:316-21.

4. Hull MC, Morris CG, Pepine CJ, Mendenhall NP. Valvular dysfunction and carotid, subclavian, and coronary artery disease in survivors of Hodgkin lymphoma treated with radiation therapy. JAMA. 2003;290:2831-7.

5. Poulin F, Seminov A, Romeo P, Demers P, Pressacco J, Basmadjian A. Extensive radiation-induced heart disease in an adult patient treated for lymphoma as a child. Can J Cardiol. 2011;27:390.e1-4.

6. Crestanello JA, McGregor CGA, Danielson GK, Daly RC, Dearani JA, Orszulak TA, et al. Mitral and tricuspid valve repair in patients with previous mediastinal radiation therapy. Ann Thorac Surg. 2004;78:826-31.

7. Park CH, Suri RM, Burkhart HM, Greason KL, Dearani JA, Schaff HV, et al. Identifying patients at particular risk of injury during repeat sternotomy: analysis of 2555 cardiac reoperations. J Thorac Cardiovasc Surg. 2010;140:1028-45.

8. Gongora E, Dearani JA, Orszulak TA, Schall HV, Zhou L, Sundt TM III. Tricuspid regurgitation in patients undergoing pericardiectomy for constrictive pericarditis. Ann Thorac Surg. 2008;85:163-71.
9. Meng X, Ao L, Song Y, Babu A, Yang X, Wang M, et al. Expression of functional toll-like receptors 2 and 4 in human aortic valve interstitial cells: potential roles in aortic valve inflammation and stenosis. Am J Physiol Cell Physiol. 2008;294: C29-35.

10. Lee JH, Meng X, Weyant MJ, Reece TB, Cleveland JC Jr, Fullerton DA. Stenotic aortic valves have dysfunctional mechanisms of anti-inflammation: implications for aortic stenosis. J Thorac Cardiovasc Surg. 2011;141:481-6.

11. Williams JR, Zhang Y, Zhou H, Gridley DS, Koch CJ, Dicello JF. Tumor response to radiotherapy is dependent on genotype-associated mechanisms in vitro and in vivo. Radiother Oncol. 2010;5:71.

12. McGale P, Darby SC, Hall P, Adolfsson J, Bengtsson NO, Bennet AM, et al. Incidence of heart disease in 35,000 women treated with radiotherapy for breast cancer in Denmark and Sweden. Radiother Oncol. 2011;100:167-75.

13. Hooning MJ, Botma A, Aleman BMP, Baaijens MHA, Bartelink H, Klijn JGM, et al. Long-term risk of cardiovascular disease in 10-year survivors of breast cancer. J Natl Cancer Inst. 2007;99:365-75.

14. Mitra AK, Singh RK, Krishna M. MAP kinases: differential activation following in vivo and ex vivo irradiation. Mol Cell Biochem. 2007;294:65-72.

15. Jelonek K, Walaszczyk A, Gabrys D, Pietrowska M, Kanthou C, Widlak P. Cardiac endothelial cells isolated from mouse heart: a novel model for radiobiology. Acta Biochim Pol. 2011;58:397-404.

16. Ryu SY, Do SH, Chung JY, Kim TH, Kim SH, Choi CY, et al. Activation of MAP kinases during progression of radiation-induced pneumonitis in rats. Hum Exp Toxicol. 2010;30:876-83.

17. Yang X, Meng X, Su X, Mauchley DC, Ao L, Cleveland JC Jr, et al. Bone morphogenic protein 2 induces Runx 2 and osteopontin expression in human aortic valve interstitial cells: role of Smad1 and extracellular signal-related kinase 1/2. J Thorac Cardiovasc Surg. 2009;138:1008-15.

18. Jinlian L, Yinbin Z, Chunbo W. p38 MAPK in regulating cellular responses to ultraviolet radiation. J Biomed Sci. 2007;14:303-12. 